Was ist der geeignete Maßstab für die Umweltleistungsmessung?

\section{Öko-Effizienz und Öko-Effektivität}

\author{
Trotz vieler Bemühungen der Unternehmen bleibt ihre Umweltleistung noch \\ weitgehend im Unscharfen. Woran läßł sich ihre "environmental performance" \\ messen? Was hat es mit dem vielbeschworenen Begriff der "Öko-Effizienz" \\ auf sich? Für eine angemessene Beurteilung müssen die Umweltwirkungen in \\ der Produktnutzungsphase sowie konterkarierende Marktwachstumseffekte \\ einbezogen werden. Dies wird am Beispiel Hausbau illustriert.
}

$\mathrm{E}$ Von Volker Stablmann und Jens Clausen tliche Unternehmen untersuchen heute ihre Umweltschwachstellen, entwickeln daraus Umweltprogramme und lassen $\mathrm{ihr}$ Umweltmanagementsystem schließlich nach der EG-Öko-Audit-Verordnung (EMAS) zertifizieren. Dies geschieht oft mit beachtlichem Umweltengagement, erstaunlicher Breite und Tiefe der Schwachstellenanalyse und einer kritischen Auseinandersetzung mit den Schwierigkeiten der Bewertung und Darstellung der Umweltleistung. Je nach Bilanzierungsgrenzen werden dabei mehr oder weniger unternehmensinduzierte Umweltbelastungen einbezogen. Da in der derzeitigen EMAS-Fassung im wesentlichen noch der enge Standortbezug im Vordergrund steht, werden regelmäßig wichtige, vom Gesamt-Unternehmen beeinflußbare Umweltbelastungen ausgeklammert. Hierzu gehört oft der produktbezogene Umweltschutz, für den nicht der Standort sondern eine zentrale Entwicklungsabteilung zuständig ist. Aber auch viele KMU entscheiden sich in Anbetracht der verwaschenen Formulierungen zur Produktverantwortung dafïr, bei den Produkten und der strategischen Planung zunächst alles so zu lassen, wie es ist. Der Validierung steht ein solches Vorgehen im Allgemeinen nicht im Wege.

Beziiglich der Realisierung wirksamer Umweltentlastungen wirft dies aber ein doppeltes Problem auf:

- erstens sind, wie aus Produktökobilanzen bekannt ist, die größten Umweltbelastungen manchmal mit der Produktnutzungsphase und nicht mit der Herstellung verbunden,

zweitens werden Fortschritte der Öko-Effizienz in der Herstellung u.U. durch vergrößerte Absatzzahlen oder intensivere Produktnutzung wieder aufgezehrt.

Im letzteren Fall bleibt zumindest unklar, welcher Anteil der Verantwortung für Marktwachstum und Nutzungsausmaß fairerweise dem Hersteller zuzurechnen ist und welcher Anteil ggf. auf die Abnehmer abgeschoben werden kann. Mit Blick auf konkurrierende Anbieter, Produkte und Funktionen bleibt weiter unklar, ob Absatzwachstum zwingend mit höherer Umweltbelastung verbunden ist oder sogar, bei Verdrängung weniger öko-effizienter Produkte vom Markt, zur wirksamen Umweltentlastung beiträgt.

Um Maßstäbe aufzustellen, an denen die Umweltleistung eines Unternehmens gemessen werden kann, ist es wichtig zwischen den Managementpotentialen, die ein Unternehmen zur fundierten Analyse und Beseitigung seiner ökologischen Schwachstellen aufbaut und der Beurteilung der tatsächlichen Umweltentlastung durch Kriterien der Öko-Effizienz und Öko-Effektivität zu trennen. Ein laufendes Forschungsprojekt zielt auf die Implementation eines in diesem Sinne vollständigen Meßsystems (1).

\section{- Effizienz versus Effektivität}

Der Bereich Managementpotentiale ist bereits Gegenstand der internen bzw. externen Umweltbetriebsprüfung nach EMAS. Neben einer formalen Prïfung der Anforderungen von EMAS oder ISO $14.001 \mathrm{muß}$ aber auch überprifft werden, ob das Umweltmanagementsystem ,lebt“ und nicht nur in Formalismen, Handbüchern, Ablagen erstarrt. Wichtig sind z.B. die Werthaltungen der Unternehmensleitung, die Unternehmenskultur, die Innovationsfähigkeit und die Art der Mitarbeiterpartizipation. Die Beurteilung der Potentiale für gutes Umweltmanagement kann dabei nicht separat von anderen Unternehmensbereichen gesehen werden. Ähnlich der Sichtweise des Qualitätsmanagements wird nach Grundfähigkeiten gefragt, die sich auf eine Pluralität von Zielen auswirken (2).

Im Bereich der Ergebnisbeurteilung sollte zwischen Öko-Effizienz und Öko-Effektivität unterschieden werden! In Anlehnung an die betriebs- wirtschaftliche Terminologie ist unter Effizienz die Wirksamkeit von Strukturen und Aktivitäten, das Wirkungsverhältnis zwischen Input und Output, zwischen Kosten und Leistung oder vice versa Ertrag und Aufwand (Wirtschaftlichkeit) zu verstehen (3).

Letzendlich maßgeblich für die Beurteilung der Umweltleistung ist allerdings die ÖkoEffektivität. Das kann im Sinne der heutigen Managementlehre so interpretiert werden, ob überhaupt die richtigen Ziele gesetzt werden, um im globalen Betrachtungsrahmen Umweltentlastungen erzielen zu können. Dies wiederum hängt entscheidend von einer umfassenden und ehrlichen Analyse der wichtigen Umweltauswirkungen und der richtigen strategischen Ausrichtung in Umweltpolitik und Umweltzielsetzungen ab. Öko-Effektivität steht aber auch dafür, ob diese Ziele dann erreicht wurden.

Effizienz ist damit eher Mittel zum Zweck, trägt mehr instrumentellen, operativen Charakter. Bei der Effektivität stehen aber die Formal- oder Sachziele selbst zur Debatte. Effektivität trägt demnach einen stark normativen Charakter (,Was sollen wir produzieren? Wie dürfen wir uns entwickeln?"*), während die Effizienz der bestmöglichen Erfüllung eines gegebenen Zielkatalogs dient.

Umweltkennzahlen, die der zielorientierten Steuerung der Umweltschutzaktivitäten dienen, müssen demnach zwischen öko-Effizienz und Öko-Effektivität trennen, z.B. in:

monetäre Kennzahlen der Öko-Effizienz (z.B. Abfallwirtschaftskostenanteil, Kostenanteil Energieträger),

- reale Kennzahlen der Öko-Effizienz (z.B. Materialeinsatz pro Produkt, spezifischer Endenergieverbrauch pro Beschäftigten),

- monetäre Kennzahlen der Öko-Effektivität (z.B. Materialeinsatz pro Wertschöpfung, Umsatzanteil Solarsysteme) und

- reale Kennzahlen der Öko-Effektivität (z.B. gesamter Energieverbrauch im Produktlebenszyklus, absoluter Energieverbrauch, Materialanteil erneuerbarer Rohstoffe)

Es ist anzunehmen, daß ein gut strukturiertes Umweltkennzahlengerüst durchaus in der Lage ist, Öko-Effizienz und öko-Effektivität operational nachprüfbar zu belegen. Ziel der Forschungsarbeit ist, beispielhaft in den Branchen Hausbau und Getränke ein Umweltkennzahlensystem zu entwickeln, welches diesem Anspruch gerecht wird. 


\section{Das Beispiel Hausbau}

Auch im Hausbau orientieren sich die meisten Umweltziele vorliegender Umwelterklärungen an der Optimierung der Herstellung; nur vereinzelt finden sich meist materielle Ziele zur ökologischen Optimierung der Produkte. Zur Erweiterung des Blickwinkels auf wichtige Umweltfragen wurden, wie auch im Entwurf der DIN/EN/ISO 14.031 ,Leitlinien Umweltleistungsbewertung“ empfohlen (4), die Standpunkte „,interessierter Kreise“ in die Zielfindung einbezogen. Ergebnis war eine starke Ergänzung der wichtigen Umweltfragen des Standorts um Umweltfragen in der Produktlinie und hier nicht nur zum ,materiellen“ Produkt sondern auch zu Fragen von Funktion und Bedürfnissen.

Für den Bau von Einfamilienhäusern wichtige, funktionsbezogene Kriterien sind z.B. die Nutzungsintensität, die durch flexible Grundrisse gefördert werden kann, oder die geschickte Optimierung der Lebensdauer, die Nutzungsrhythmen der BewohnerInnen (z.B. Zeiten der Kindererziehung) und technische Innovationszyklen (z.B. der Haustechnik) berücksichtigt. Durch Fortschritte in diesen Bereichen könnten gerade die besonders bedeutsamen Umweltbelastungen aus der Produktnutzungsphase wirksam reduziert werden.

Im Rahmen des auf Unternehmen des Hausbaus bezogenen Projektes erschien problematisch, daßmehrere der aufgezeigten Optmierungsnotwendigkeiten eigentlich nur auf der Ebene der Stadtplanung oder zumindest gemeinsam mit Bauträgern für ganze Baugebiete beeinflußt werden können. Hierzu gehören z.B. Fragen des „gebauten Verkehrs“. Auch Optimierungspotentiale durch Mehrfachnutzung von Heizsystemen, Freizeiträumen oder Geräten sind im Bau einzelner Einfamilienhäuser kaum realisierbar, können aber in Baugebieten erschlossen werden.

Dies macht Systemlösungen und damit Kooperationen in der Produktlinie aber auch solche mit Akteuren der kommunalen Planung notwendig. Optimal öko-effektiver Hausbau ist also im klassischen Verkauf eines Einfamilienhauses an einen Einzelbauherren u.U. gar nicht zu erzielen.

\section{- Markfwachstum und Öko-Effektivität}

Relative Verbesserungen der Öko-Effizienz werden, vom Unternehmen aus gedacht, oft durch Wachstumseffekte wieder aufgezehrt. Die Auseinandersetzung mit Wachstum gerät damit ins Zentrum der ökologischen Strategiefindung. Im Rahmen der Vorstudie konnten einige Kriterien gefunden werden, die eine Beurteilung des Unternehmenswachstums erlauben könnten.

1. Ist das Produkt relativ öko-effizienter als Produkte gleicher Zabl und Funktion, die es ersetzt?

Das Produkt höherer Öko-Effizienz wird eine gleiche Zahl „schlechterer“ Produkte vom Markt verdrängen und so letztlich zu einer absoluten Entlastung der Umwelt führen. Die höhere Öko-Effizienz wird effektiv umweltentlastend wirksam (Beispiel: Brennwertkessel ist öko-effizienter als eine herkömmliche Heizung). Unterschiedliche Varianten dieses Agierens im gleichen Markt werden auch von Wüstenhagen et al. in ihrer „Landkarte des ökologischen Massenmarktes" beschrieben (5). Dieses Unternehmenswachstum wäre also im Sinne einer ökologiegerechten Änderung der Produktionsstrukturen positiv zu beurteilen.

2. Erfüllt das neue Produkt eine neue, zusätzliche Funktion oder fördert es den Umfang der Nutzung?

Das Wachstum des Produktmarktes würde hier keine anderen, weniger öko-effizienten Produkte verdrängen sondern zu zusätzlichem Konsum führen (Beispiel: Tamagotchi). Auch die absolute Zunahme der Nutzung steigert die hiervon abhängigen Verbräuche (Beispiel: unfunktional großes Wohnzimmer). Der Ressourcenverbrauch wird in beiden Fällen absolut steigen. Dies ist ethisch nur dann gutzuheißen, wenn durch die zusätzliche Umweltbelastung $\mathrm{zu}$ Lasten der kommenden Generation weitere, unverzichtbare Bedürfnisse der gegenwärtigen Generation befriedigt werden. Orientierung kann hier vielleicht die Beantwortung der Frage nach der Generalisierbarkeit bieten - die Grenzen der ansonsten hilfreichen Landkarte des ökologischen Massenmarktes sind aber deutlich überschritten.

3. Die Frage nach dem ,kategorischen Imperativ" Kant's stellen.

Kann die Frage: „Wäre es nachhaltig, wenn jeder sich so verhalten würde, bzw. dieses Produkt konsumierte?"“ mit ja beantwortet werden, dann ist das Produkt ökologisch sinnvoll (6). Der Bau von $160 \mathrm{~m}^{2}$-Einfamilienhäusern ist z.B. kaum generalisierbar, wogegen die Dienstleistung ,energetische Renovierung von Wohnung oder Haus" weltweit verallgemeinerbar wäre.

\section{- ISO 14.031 nicht weitreichend genug}

Das Projekt baut auf dem Normentwurf der DIN/EN/ISO 14.031 zur Umweltleistungsbewertung auf. Bei der Anwendung im Hausbau geht das Projekt aber mit konsequenter Produkt-, Funktions- und Marktorientierung auch über diesen hinaus. Daher ist auch die Kritik und Ergänzung des Normentwurfes ein Ziel des Projektes.

Als Orientierungsgrößen der Öko-Effektivität über den Normentwurf hinaus scheint es wichtig, sich mit Wachstum und seinen Grenzen auseinanderzusetzen, den Gebrauchsnutzen der Produkte mit Blick auf Grundbedürfnisse und das ,gute Leben“ zu optimieren sowie die klassischen Ziele des Umweltschutzes im operativen und besonders auch im strategischen Management konsequent weiter zu verfolgen.

\section{Anmerkungen}

(1) Die Autoren bearbeiten hierzu das Projekt „Beurteilung der Umweltleistungsfähigkeit von Unternehmen anhand von Kriterien seiner Managementpotentiale, der Öko-Effizienz und der Öko-Effektivität", gefördert von der Deutschen Bundesstiftung Umwelt.

(2) Vgl. Pfriem, R.: Vom Umweltmanagement zur auch ökologischen Entwicklungstähigkeit von Unternehmen - Die zweite Phase ökologischer Unternehmenspoltik; erscheint in: Bellmann, K:: Umweltmanagement, Wiesbaden 1999.

(3) Dies ist im wesentlichen der Kerngedanke zur Umweltentlastung, wie er im Brundtland-Bericht, in Schmidtheiny's „Kurswechsel" oder E.U. v. Weizsäcker/ A.Lovins/ H.Lovins "Faktor 4" zum Ausdruck kommt.

(4) Vgl. DIN/EN/ISO 14.031 Leitlinien Umweltleistungsbewertung, Berlin 1998.

(5) Vgl. Wüstenhagen, R./ A. Meyer/ A. Villiger: Die Landkarte des ökologischen Massenmarktes. In: Ökologisches Wirtschaften 1/1999, S. 27-29.

(6) Vgl. auch Hössle, V.: Bedarf und Bedürnis. In: Future das Hoechst Magazin 2/98.

\section{Die Autoren}

Prof. Dr. Volker Stahlmamn lehrt an der FH Nürnberg Material-und Fertigungswirtschafi sowie Umweltmanagement; Jens Clausen ist wissenschaftlicher Mitarbeiter am löW.

Kontakt: Volker Stahlmann, FH Nürnberg, FB BW, Bahnhofstr. 87, 90402 Nürnberg,

Tel. 0911/5880-2870, Fox -6870 ,

E-mail: Volker.Stahlmann@th-nuernberg.de; Jens Clausen, IÖW-Projektbüro Hannover, Hausmannsir. 9-10, 30159 Hannover, Tel. 0511/ 16403-44, Fax -91, E-mail: Jens.Clausen@hannover.ioew.de 
(c) 20I0 Authors; licensee IÖW and oekom verlag. This is an article distributed under the terms of the Creative Commons Attribution Non-Commercial No Derivates License (http://creativecommons.org/licenses/by-nc-nd/3.o/), which permits unrestricted use, distribution, and reproduction in any medium, provided the original work is properly cited. 